\title{
Reaction Time Significance: Dropping Metersticks, the Stroop Effect \& Implicit Bias Tests
}

\author{
Laurel L. Hester
}

\author{
Keuka College, Division of Natural Sciences and Mathematics, 141 Central Ave, Keuka Park \\ NY 14478 USA \\ (lhester@keuka.edu)
}

\begin{abstract}
The meterstick drop is a tried and true lab exercise for measuring reaction time - often presented in an inquirybased format with students testing their own reaction-time hypotheses. In this lab, comparison of meterstickdrop results with dot-making results facilitates discussion of how and why reaction time varies by activity. This point is then emphasized by having participants complete the Interactive Stroop Effect Experiment by Eric Chudler (available online from the University of Washington's "Neuroscience for Kids" website). The well-studied Stroop effect test uses the time a person takes to read a word list aloud as a proxy measure for cognitive processing load. When a color word's meaning and ink-color conflict, the reading-aloud task time takes longer. The Stroop experiment's use of read-aloud task time to investigate cognitive processing provides an introduction, in turn, to Harvard's Project Implicit ${ }^{\circledR}$. Participation in one of that site's less controversial implicit bias tests is the basis for a final discussion of what reaction times reveal and how experimental design of reaction-time tests can be optimized.
\end{abstract}

Keywords: reaction time, Stroop effect, implicit bias, inquiry-based learning

\section{Introduction}

This sequence of reaction time tests is intended to sequentially build student understanding of neural pathway complexity and how our brain's automatic interpretation of reality affects our interactions with our environment. As with many inquiry-based laboratory exercises, it is best to let students explore and discover the key findings on their own. This laboratory activity is very flexible in the amount of emphasis and detail placed on various components. I like using the initial ruler-catch reaction time activity in the first laboratory meeting of the semester to introduce basic nervous system vocabulary and structures. Because it is a fun, dynamic lab, it also serves well as an icebreaker activity during the first lab. Students then complete the Stroop effect and implicit bias investigations later in the semester after a unit on optical illusions. However, a streamlined version of the lab that could be performed in one longer lab period is presented here. This lab activity can easily be modified for use in a variety of college-level courses such as Introductory Biology, Human Biology, Anatomy and Physiology or Neurobiology. The amount of technical vocabulary introduced and the level of neural pathway detail presented can be tailored to the course level.

\section{Measuring Reaction Time}

The initial laboratory activity compares two simple reaction time measurements. When combined with a detailed review of vocabulary and nervous system structures, the activity takes about two hours. It can be shortened by omitting the vocabulary and structure review or extended if students actually perform the additional reaction time experiments they design.

\section{Dropping Metersticks}

Many students have estimated their reaction time by having a partner drop a meter stick and measuring how many centimeters it falls before they catch it. Example write-ups of these activities can be easily found in any Google search (e.g. see Yunas, 2012). Often this activity is paired with an introduction to experimental design and followed by students brainstorming various hypotheses they could test. The novel twist here is using this activity to introduce neurobiology terms such as sensory neuron, interneuron, motor neuron and/or brain structures such as optic nerve, thalamus, occipital cortex and then comparing reaction time measurements and neural pathways with a different type of reaction time measurement. 


\section{Polka-dots}

Reaction time estimates based on how many dots one can make in 5 seconds are reliably shorter. Comparing these two measures using an online paired ttest calculator provides for a rich discussion about how the two reaction time protocols differ, about use of unpaired vs. paired t-tests and about descriptive vs. inferential statistics and about experimental design. At this point, one might want to discuss hypotheses, predictions, control variables and/or even have students test hypotheses in lab (e.g. dominant vs. non-dominant hand, visual vs. auditory cues, effects of practices, etc.).

\section{Initial Take-home Messages}

In order to prepare students for the following activities, it is useful to emphasize 1) reaction times are fast 2) many neurons are involved in even very simple behaviors with many interneuron brain connections and 3) perception occurs in the brain.

\section{The Stroop Effect}

The well-studied Stroop effect (Stroop, 1935) notes that it takes a person longer to read a word list aloud when a color word's meaning and ink-color conflict (e.g. the word 'green' printed in red ink). See Macleod (2014) for an excellent review. Use of Chudler's (2015) online Stroop Effect Experiment gives students a rapid introduction to this effect, but a thorough investigation of this effect could easily take an hour or more. However, be aware that there is ongoing debate over explanations for this effect, and it is possible that there is no single simple explanation for all that occurs to cause this effect and variations in its strength. Fro example, I have noticed that this effect is much weaker or absent in international students that grew up reading a different script (e.g. Chinese characters). The Stroop experiment's use of read-aloud task time to investigate cognitive processing provides an introduction to the idea that the presence of information that conflicts with our expectations or the presence of conflicting cues slows processing time.

\section{Implicit Bias}

The Kirwan Institute for the Study of Race and Ethnicity (2015) defines implicit bias (also known as implicit social cognition) as "the attitudes or stereotypes that affect our understanding, actions, and decisions in an unconscious manner. These biases, which encompass both favorable and unfavorable assessments, are activated involuntarily and without an individual's awareness or intentional control." Harvard University's Project Implicit ${ }^{\circledR}$ has developed several "Implicit Bias Tests" that use reaction time to reveal possible implicit biases. These tests have been cited in several recent news articles and used in some "implicit bias" trainings. Based on the earlier experiences with the other reaction time tests, students hopefully approach these implicit bias tests with an open mind, an appreciation for the complexity of neural pathways and an interest in thoughtfully examining this use of a reaction time test to reveal unconscious "biases."

Note: Additional online resources including a Google Sheets data table and the ABLE mini-workshop presentation can be found in the tinyurl.com/rxtime link given in the Student Outline. 


\section{Student Outline}

\section{Objectives}

- Be able to give examples of situations where reaction time is relevant to daily activities.

- Describe the many different steps your nervous system must perform in order to complete a 'simple' behavior.

- Know about how long it takes an average person to perform a 'simple' behavior.

- Brainstorm ideas about factors that might affect reaction time.

- State a testable hypothesis and be able to propose a simple experiment to test your hypothesis.

- Consider how potentially confounding variables should be 'controlled' in experimental designs.

- Describe the Stroop Effect and provide an interpretation of its results.

- Define 'implicit bias' and explain the rationale behind using reaction time as a measure in implicit bias tests.

- Reflect on what implicit bias test results might indicate.

- Be able to discuss the relationship between experimental results, their interpretation and public discourse.

\section{Introduction}

This series of three lab activities will illustrate several key points about how your nervous system functions. First, we will consider the importance of reaction time and measure it using two simple tests. Discussion of how the nervous system functions during these two tests introduces basic nervous system concepts and vocabulary. Comparison of the two tests and statistical analysis of class results reveals several important experimental design considerations. Second, experience with the Stroop effect demonstrates potential performance costs when conflicting signals increase cognitive load. Finally, you will consider possible real-world consequences of information-processing variation after trying a controversial reaction time test.

\section{Methods and Data Collection}

\section{Measuring Reaction Time:}

1. People are different in how fast they can react to something they see. Sometimes this is important to their activity, sometimes not. What are some activities in which having a fast reaction time is important?

After sharing your ideas as a class, pay attention while your instructor works with a volunteer student to demonstrate how you can estimate your reaction time by catching a dropped meterstick.

2. What had to happen in order for our demo student to catch the ruler? (think about neurons)

Initial Thoughts (during demonstration) Additions/Modifications after Discussion

3. Each of you should catch a meterstick drop three times (record your data below).

's Eye-Hand Reaction distance

\begin{tabular}{|l|l|l|}
\hline Trial $1(\mathrm{~cm})$ & Trial $2(\mathrm{~cm})$ & Trial $3(\mathrm{~cm})$ \\
\hline & & \\
\hline
\end{tabular}

's Eye-Hand Reaction distance

\begin{tabular}{|l|l|l|}
\hline Trial $1(\mathrm{~cm})$ & Trial $2(\mathrm{~cm})$ & Trial $3(\mathrm{~cm})$ \\
\hline & & \\
\hline
\end{tabular}

Are reaction distances different between trials for one person? (yes/no) Why might this be? 
Did you and your lab partner get different averages? (yes/no)

Why might this be?

Go to tinyurl.com/343Lab1 to convert from average centimeters dropped to reaction time in seconds (time for the ruler to fall that distance).

Partner's reaction time (sec) Your reaction time $(\mathrm{sec})$ (report to closest $0.01 \mathrm{sec}$ )

4. Another way to measure reaction time is to see how fast you can make pencil dots on a piece of paper. Your partner will tell you when to start and when to stop ( 5 seconds later). Make as many separate dots as you can in that time. This activity is a bit different because your muscles need to relax before they contract again, but there are some other differences as well...

You made dots in 5 seconds. Your reaction time is 5 seconds divided by this number of dots.

Your reaction time for the dot making activity: sec (should be less than 1)

Go to the class data sheet and add your reaction times for both the ruler drop and the dot-making. If you are one of the first groups to finish, complete questions $5 \& 6$ and then return to complete your answer below once at least 10 people's data have been posted.

Does it look like there is a difference between reaction times for these two activities? Yes / No / Unsure - Explain below.

5. Based on what you have observed, your own life experience and what we have discussed today, what factors (at least 3) do you think might affect reaction time?

6. Decide on a factor you could theoretically test with the rest of your table and write out your hypothesis. In science, a hypothesis is an idea or explanation that you then test through study and experimentation using observations of the natural world.

Hypothesis:

Which variables might you want to control or keep the same during your experiment?

7. On a computer or even your phone, go to: faculty.washington.edu/chudler/java/ready.html. Read the instructions and when you are ready, click "go to the first test". When you are done reading the words aloud and click the FINISH button to see your time. Record your time below BEFORE clicking "continue experiment" to get the second word list. Click the FINISH button after you've read the second word list and record that time below.

1st wordlist time: sec; 2nd wordlist time: sec. Enter data at tinyurl.com/rxtime.

Was there a difference in your times? Yes / No (circle one) Why? (Click on 'Back to Stroop Effect' for explanation)

8. Go to: https://implicit.harvard.edu/implicit/takeatest.html (or use the link from our data sheet). Read the preliminary information and then click "proceed" or email your instructor if you have any questions. After clicking to proceed, examine the test options and consider which ones interest you, but wait for your instructor's explanation before continuing. Before next lab period, take at least one implicit bias test and submit your reflection by going to tinyurl.com/IBreflectX. You will NOT have to include your results, but DO have to answer all the questions OR complete the alternative writing assignment in order to receive full credit for this lab worksheet. 


\section{Cited References}

Chudler EH. 2015. Neuroscience For Kids - stroop effect. [accessed 2019 Oct 31]. https://faculty.washington.edu/chudler/words.html.

Stroop JR. 1935. Studies of interference in serial verbal reactions. Journal of Experimental Psychology. 18:643-662.

Take a Test. [accessed 2019b Oct 31]. https://implicit.harvard.edu/implicit/takeatest.html. 


\section{Materials}

Each pair of students will need a meterstick, pen, paper, a timer, internet access and a computer.

\section{Notes for the Instructor}

This laboratory exercise is very customizable and leads to great discussion. It works well to go back and forth between letting students work on their own and pausing them to go over their answers as a group. For question 2, I typically write on the board as students report their ideas, leaving space for the points I want to elicit. My final list often looks something like:

- Recognize that ruler is falling

- receptors respond

- neurons fire (optic nerve)

- relay in thalamus

- $1^{\text {st }}$ conscious detection in occipital lobe (visual cortex)

- additional processing in parietal lobe

- decide to catch

- frontal lobe (pre-frontal cortex)

- $\quad$ signal to move

- $\quad$ frontal lobe (motor cortex)

- neurons carry info down spinal cord

- $\quad$ other neurons carry info out to periphery

- $\quad$ tell muscles to contract

Students often omit the "decide to catch" step. Other common mistakes are misreading the dot reaction time calculation (and flipping the fraction) and, of course, just not reading the instructions. For the online Stroop and Implicit Bias tests, I often demonstrate first. For the Implicit Bias tests, I let them know they can give fake answers to the demographic questions if they want. Because results are sometimes sensitive, I often start by sharing my results that I have some "bias" against women in science and this lets us discuss how we are influenced by what we see in the society around us. I usually send students home to take the implicit bias tests since the results may be sensitive for some of the tests. After students reflect on their results, it can be fascinating to have students do a web search for "implicit bias" to see how the topic is covered in the news.

\section{Cited References}

Chudler EH. 2015. Neuroscience For Kids - stroop effect. [accessed $2019 \quad$ Oct 31]. https://faculty.washington.edu/chudler/words.ht $\mathrm{ml}$.

Kirwan Institute for the study of race and ethnicity. 2015. Understanding Implicit Bias. [accessed 2019 Oct 31].

http://kirwaninstitute.osu.edu/research/understan ding-implicit-bias/.

MacLeod CM. 2014. The Stroop Effect. In: Luo R, editor. Encyclopedia of Color Science and Technology. Berlin, Heidelberg: Springer. p. 1-6. [accessed 2019 Oct 31]. https://doi.org/10.1007/978-3-64227851-8_67-1.

Stroop JR. 1935. Studies of interference in serial verbal reactions. Journal of Experimental Psychology. 18:643-662.

Take a Test. [accessed 2019b Oct 31]. https://implicit.harvard.edu/implicit/takeatest.ht $\mathrm{ml}$.

Yuhas D. Speedy Science: How Fast Can You React? Scientific American. [accessed 2019 Oct 31]. https://www.scientificamerican.com/article/bring -science-home-reaction-time/..

\section{Acknowledgments}

I would like to thank Regina McClinton and my son Alex Raguso for introducing me to these implicit bias tests.

\section{About the Authors}

Laurel Hester has taught biology for eight years at Keuka College and previously taught biology labs at Cornell University, the University of South Carolina and the SC Governor's School for Science and Mathematics. After a brief stint as Director of Keuka College's Center for Teaching and Learning, Laurel was tempted to cross over to academic administration and is currently serving as Assistant Provost. She loves working with faculty colleagues across disciplines, but misses teaching lots of biology labs. 


\section{Mission, Review Process \& Disclaimer}

The Association for Biology Laboratory Education (ABLE) was founded in 1979 to promote information exchange among university and college educators actively concerned with teaching biology in a laboratory setting. The focus of ABLE is to improve the undergraduate biology laboratory experience by promoting the development and dissemination of interesting, innovative, and reliable laboratory exercises. For more information about ABLE, please visit http://www.ableweb.org/。

Advances in Biology Laboratory Education is the peer-reviewed publication of the conference of the Association for Biology Laboratory Education. Published articles and extended abstracts are evaluated and selected by a committee prior to presentation at the conference, peer-reviewed by participants at the conference, and edited by members of the ABLE Editorial Board. Published abstracts are evaluated and selected by a committee prior to presentation at the conference.

\section{Citing This Article}

Hester LL. 2020. Reaction time significance: Dropping metersticks, the Stroop effect \& implicit bias tests. Article 37 In: McMahon K, editor. Advances in biology laboratory education. Volume 41. Publication of the 41st Conference of the Association for Biology Laboratory Education (ABLE). https://doi.org/10.37590/able.v41.art37

Compilation (C) 2020 by the Association for Biology Laboratory Education, ISBN 1-890444-17-0. All rights reserved. No part of this publication may be reproduced, stored in a retrieval system, or transmitted, in any form or by any means, electronic, mechanical, photocopying, recording, or otherwise, without the prior written permission of the copyright owner.

ABLE strongly encourages individuals to use the exercises in this volume in their teaching program. If this exercise is used solely at one's own institution with no intent for profit, it is excluded from the preceding copyright restriction, unless otherwise noted on the copyright notice of the individual chapter in this volume. Proper credit to this publication must be included in your laboratory outline for each use; a sample citation is given above. 